\title{
RADIOTHERAPY FOR METASTATIC BREAST CANCER IN MEXICO: RESULTS FROM THE 2015 NATIONAL SURVEY
}

\author{
Nora Álvarez-Águila ${ }^{1 *}$, Hilary Cook $^{2}$, Diddier Prada 3,4 , Aida Mota-García ${ }^{1}$, Luis A. Herrera ${ }^{4}$, \\ Alejandro Mohar-Betancourt ${ }^{4}$, Abelardo Meneses-García ${ }^{4}$ and Felicia M. Knaul ${ }^{5-8}$
}

${ }^{1}$ Department of Radiotherapy, Instituto Nacional de Cancerología, Mexico City, Mexico; ${ }^{2}$ Harvard Global Equity Initiative, Department of Global Health \& Social Medicine, Harvard Medical School, Boston, MA, USA; ${ }^{3}$ Department of Environmental Health, Harvard School of Public Health, Boston, MA, USA; ${ }^{4}$ Biomedical Research Unit in Cancer, Instituto Nacional de Cancerología and Instituto de Investigaciones Biomédicas, Universidad Nacional Autónoma de México, Mexico City, Mexico; ${ }^{5}$ Department of Public Health Sciences, University of Miami Miller School of Medicine, Coral Gables, Florida, USA; ${ }^{6}$ University of Miami Institute for Advanced Study of the Americas, Coral Gables, Florida, USA; ${ }^{7}$ Programa Universalidad y Competitividad en Salud, Fundación Mexicana para la Salud, Mexico City, Mexico; ${ }^{8}$ Asociación "Tómatelo a Pecho", Mexico City, Mexico

\begin{abstract}
Background: Radiation therapy is a keystone to improve survival and quality of life in breast cancer patients. In Mexico, however, scarce information is available on the obstacles faced by radio-oncologists to provide appropriate treatment. Objective: To determine the most frequent issues faced by physicians to provide radiation therapy for metastatic breast cancer in Mexico. Methods: A survey of 16 multiple-choice questions to be answered electronically by 167 radio-oncologists currently working in Mexico was designed and thereafter analyzed for differences between private and public practices, based on the responses from the surveyed participants. Results: $98.5 \%$ of surveyed responders attended patients with breast cancer. We observed a significant difference between private vs. public practice for the main difficulties in providing radiation therapy, with an increased frequency $(85.8 \%)$ of "treatment cost by itself" in private practice vs. $50.7 \%$ in public practice $(p<0.05)$. Significant differences were observed in the "Time to initiate treatment" question, with "Less than one week" as the response in $86 \%$ of those physicians in private practice vs. $50 \%$ for those in public practice $(p<0.001)$. Conclusions: Using a survey targeted at radiooncologists, we analyzed the most important obstacles for accessing radiation therapy for metastatic breast cancer in Mexico. This information may be useful for healthcare decisions related to radiation therapy in women with breast cancer in Mexico. (REV INVES CLIN. 2017;69:11-9)
\end{abstract}

Key words: Breast cancer. Mexico. National survey. Palliative care. Radiation therapy.

Corresponding author:

*Nora Patricia Álvarez-Águila

Department of Radiotherapy

Instituto Nacional de Cancerología

Av. San Fernando, 22

Col. Sección XVI, Del. Tlalpan

C.P. 14080, Ciudad de México, México

E-mail: noraalvarezaguila@yahoo.com.mx

Received for publication: 06-09-2016

Accepted for publication: 05-12-2016 


\section{INTRODUCTION}

Breast cancer (BC) is the most common malignant neoplasia worldwide, with 1.7 million new cases diagnosed every year ${ }^{1-3}$. Remarkably, $62 \%$ of deaths due to breast cancer occur in less developed countries, where $33 \%$ of the cases correspond to women between 15 and 49 years of age ${ }^{1-3}$. Lack of access to radiotherapy is a limiting factor for improving the quality and efficacy of care worldwide ${ }^{4}$.

Mortality for $\mathrm{BC}$ has been trending upward in Mexico for several decades ${ }^{5-7}$. Given the increased frequency of advanced disease at diagnosis in Mexico and the lack of radiotherapy units, $85 \%$ of cases attended in third level reference hospitals undergo mastectomy ${ }^{1}$.

Radiation therapy is a keystone in BC therapy from early stages until advanced local disease, and also is essential for metastatic disease. Currently, patients with $B C$ in early stages must be treated using conservative surgery plus radiotherapy ${ }^{1-3,8}$. For patients with advanced disease, radiotherapy is also a key component in the treatment and it is typically indicated after mastectomy. Radiotherapy is also used for the treatment of breast cancer metastasis, including bone, brain, and soft-tissue metastasis ${ }^{1-3,9}$. Radiation therapy also has favorable results in palliative care, relieving symptoms earlier and demonstrating functional recovery in a more efficient manner compared to other therapies. Additionally, palliative radiotherapy for bone metastasis in one fraction treatment is cost-effective and better than pain medication and chemotherapy ${ }^{5-7,10}$.

Although Mexico offers universal health system coverage for every women diagnosed with $\mathrm{BC}$, including the best available therapies (e.g. HER2-inhibitors), patients need to overcome several obstacles to accessing effective treatment, including long commutes, lack of places to stay near to hospitals, and other costs associated with treatment in units located far away from their homes. Most of these difficulties are frequently derived from low socioeconomic status ${ }^{1,11}$. To date, precise information about the issues faced by patients and radio-oncologists to offer appropriate radiation therapy in Mexico is not available. To address this lack of data, we developed a nationally targeted survey to understand these issues from the perspective of the radio-oncologist, and to determine the most frequent obstacles related to radiation therapy for metastatic
BC patients and palliative care in Mexico. A more precise understanding of radiation therapy in Mexico could potentially improve the operative capacity in radiotherapy units.

\section{MATERIALS AND METHODS}

\section{Study participants}

Radio-oncologists working in Mexico were identified using publicly available data sources and were contacted by e-mail and/or phone calls. Using openly available Internet sources, such as Google and Linkedin, and applying a search strategy ("radiotherapy" OR "radiotherapist" OR "therapy" AND "radiation" AND "Mexico"), we identified potential participants for the study. Additionally, we attempted to contact all specialized radio-oncologists who had worked in the Instituto Nacional de Cancerología over the previous 20 years.

The physicians were invited to participate and asked about the conditions under which they offer radiotherapy for BC. In order to be included, participants had to access the survey website and answer all the questions. The study protocol and survey were approved by the Institutional Review Board (IRB) of Harvard Medical School (IRB15-0226).

\section{Survey}

The survey consisted of 16 multiple-choice questions. The survey was in English (as approved by Harvard IRB), and responses were analyzed using the Qualtric package (American University, Washington, D.C., USA) available from the Harvard Medical School. This software creates a unique, blind ID for each participant. The survey included questions on:

- percentage of patients who have breast cancer;

- frequency of metastatic breast cancer among treated patients;

- percentage of patients with breast cancer who required palliative radiotherapy;

- difficulties in starting radiotherapy;

- difficulties in ending radiation therapy; 
- time for starting treatment;

- dose-fractionation schemes for brain and bone metastasis;

- percentage of patients who benefit from palliative radiotherapy;

- improvement of symptoms with radiation therapy.

Finally, there was an open question on how access to radiation therapy can be improved in Mexico. The answers to the questionnaire applied are shown in Supplementary Figure 1.

\section{Statistical analysis}

Differences in proportions across individual participants and by the type of medical practice (private, public, or private and public) were compared using the normal approximation, which consists of calculating a weighted sum of squared deviations between the observed proportions in each group and the overall proportion for all groups ${ }^{12}$. The $p$ values $<0.05$ were considered significant. Data were analyzed using the $\mathrm{R}$ statistical software package (version 3.2.1, The R Foundation for Statistical Computing, Vienna, Austria).

\section{RESULTS}

\section{Target survey population}

Of the 204 radio-oncologists identified ${ }^{5}$, the survey was applied only to those in whom contact by e-mail was possible (total, 167 radio-oncologists); 68 completed the survey, for a response rate of $40.7 \%$.

From the population surveyed, $21 \%$ attend patients in a private office only, $15 \%$ in public hospitals, and $65 \%$ in both (Table 1 ). A complete description of responses by type of medical practice is presented in table 2.

\section{Frequency of metastatic breast cancer treated}

Only one of the surveyed radio-oncologists from public practice attended $B C$ patients exclusively, while the remainder treated $\mathrm{BC}$ and other types of cancer
Table 1. Characteristics of population surveyed in the Radiotherapy 2015 National Survey for metastatic breast cancer

\begin{tabular}{lc}
\hline Variable & $\mathbf{n}$ or $\%$ \\
\hline Number of specialists & \\
Estimated* & 167 \\
Surveyed & 68 \\
Type of practice & \\
Public & $15 \%$ \\
Private & $21 \%$ \\
Public and private & $65 \%$ \\
Types of tumors treated & \\
Breast cancer only & $1 \%$ \\
Breast cancer and other tumors & $99 \%$ \\
\hline
\end{tabular}

*Based on the population estimated for surveying.

(Table 1). A borderline significance for differences in the type of cancer patients attended was observed for those radio-oncologists in public practice $(p=0.05)$, but not for those in private practice.

\section{Proportion of breast cancer among radiotherapy patients}

In most of the surveyed specialists (76\%), more than $40 \%$ of the attended patients corresponded to BC. Among the radio-oncologists in public practice, less than $20 \%$ of their patients had BC (Table 2 ).

\section{Proportion of patients with breast cancer who need palliative radiotherapy}

Almost one-third of the surveyed physicians responded that $20-39 \%$ of patients with $B C$ required palliative care, whereas $\sim 10 \%$ of the radio-oncologists answered that at least $80 \%$ of their patients required palliative radiotherapy. No differences were observed between private and public practice.

\section{Difficulties in providing radiation therapy}

The results showed that income represented a key barrier for accessing radiotherapy (39\%), with no statistically significant differences between private and public practice (Table 2 ). In public practice, the waiting time to begin treatment was the primary difficulty (31\%), followed by the lack of hospital facilities (23\%). For radio-oncologists in private practice, the 
Table 2. Number and percentage of responses from 2015 National Survey: Radiotherapy for metastatic breast cancer in Mexico

Type of medical practice

\begin{tabular}{|c|c|c|c|c|c|c|c|c|c|c|}
\hline \multicolumn{2}{|c|}{ All } & \multicolumn{3}{|c|}{ Private } & \multicolumn{3}{|c|}{ Public } & \multicolumn{3}{|c|}{ Private and Public } \\
\hline$n$ & $(\%)$ & $\mathrm{n}$ & $(\%)$ & $\begin{array}{c}p \\
\text { value }^{\dagger}\end{array}$ & $n$ & (\%) & $\begin{array}{c}p \\
\text { value }^{\dagger}\end{array}$ & $\mathrm{n}$ & $(\%)$ & $\begin{array}{c}p \\
\text { value }^{\dagger}\end{array}$ \\
\hline
\end{tabular}

Types of tumors treated Breast cancer only

$\begin{array}{rrrr}1 & (1.5) & 0 & (0.0) \\ 67 & (98.5) & 14 & (100.0)\end{array}$

Breast cancer and other primary tumors

Any other (except breast

$\begin{array}{llll}0 & (0.0) & 0 & (0.0)\end{array}$

(0.0) $\quad 0.77$

1 (10.0)

9 (90.0)

cancer)

Frequency of patients with

breast cancer

$60-79 \%$

$40-59 \%$

$20-39 \%$

$<20 \%$

$\begin{array}{rrrr}10 & (14.9) & 0 & (0.0) \\ 16 & (23.9) & 5 & (38.5) \\ 25 & (37.3) & 7 & (53.8) \\ 15 & (22.4) & 1 & (7.7) \\ 1 & (1.5) & 0 & (0.0)\end{array}$

Frequency of palliative

therapy*

$>80 \%$

$60-79 \%$

$40-59 \%$

$20-39 \%$

$<20 \%$

$\begin{array}{rlll}7 & (10.3) & 1 & (7.1) \\ 15 & (22.1) & 2 & (14.3) \\ 13 & (19.1) & 2 & (14.3) \\ 22 & (32.3) & 4 & (28.6) \\ 11 & (16.2) & 5 & (35.7)\end{array}$

$\begin{array}{lll} & 1 & (10.0) \\ 0.15 & 2 & (20.0) \\ & 4 & (40.0) \\ & 2 & (20.0) \\ & 1 & (10.0)\end{array}$

$\begin{array}{rrr} & 9 & (20.0) \\ 0.20 & 9 & (20.0) \\ & 14 & (31.1) \\ 12 & (26.7) \\ & 1 & (2.2)\end{array}$

(0.0)

$0 \quad(0.0)$

0.05

$44 \quad(100.0)$

$0 \quad(0.0)$

0.16

Difficulties to receive

radiation therapy*

Income

Frequency of therapies

Lack of understanding

Waiting times

Lack of facilities

$\begin{array}{rrrr}41 & (38.7) & 10 & (45.5) \\ 17 & (16.0) & 3 & (13.6) \\ 12 & (11.3) & 4 & (18.2) \\ 22 & (20.8) & 1 & (4.5) \\ 14 & (13.2) & 4 & (18.2)\end{array}$

$\begin{array}{lll} & 1 & (10.0) \\ 0.28 & 3 & (30.0) \\ & 2 & (20.0) \\ & 2 & (20.0) \\ & 2 & (20.0)\end{array}$

$\begin{array}{rrr} & 5 & (11.4) \\ & 10 & (22.7) \\ 0.91 & 9 & (20.4) \\ & 16 & (36.4) \\ & 4 & (9.1)\end{array}$

0.18

Percentage of treatment

completed*
$\geq 90 \%$
$75-89 \%$
$50-74 \%$
$<50 \%$

$\begin{array}{rr}56 & (82.4) \\ 9 & (13.2) \\ 1 & (1.5) \\ 2 & (2.9)\end{array}$

(100.0)

(0.0)

(0.0)

(0.0)

Reason for stopping

treatment*

Financial

Commuting

Family support

Lack of understanding of benefits

Patients sought other options

$\begin{array}{rrrr}35 & (35.4) & 7 & (35.0) \\ 26 & (26.3) & 6 & (30.0) \\ 10 & (10.1) & 2 & (10.0) \\ 9 & (9.0) & 2 & (10.0) \\ 19 & (19.2) & 3 & (15.0)\end{array}$

$\begin{array}{rrr} & 8 & (80.0) \\ 0.29 & 2 & (20.0) \\ & 0 & (0.0)\end{array}$

$\begin{array}{rrr} & 34 & (77.3) \\ 0.81 & 7 & (15.9) \\ & 1 & (2.3) \\ & 2 & (4.5)\end{array}$

0.44

$\begin{array}{lll}0.98 & 1 & (7.7)\end{array}$

$0 \quad(0.0)$

$\begin{array}{rrr} & 24 & (36.4) \\ & 15 & (22.7) \\ & 5 & (7.6) \\ 0.18 & 6 & (9.1)\end{array}$

0.30

$16 \quad(24.2)$

From a financial perspective,

what is the principal

difficulty in providing

treatment*

Treatment cost itself

Resources for food and

$\begin{array}{rrrr}34 & (50.7) & 12 & (85.8) \\ 17 & (25.4) & 1 & (7.1\end{array}$

lodging

Patient loses his/her

11 (16.4)

$\begin{array}{ll}0 & (0.0)\end{array}$

5.8)

(7.1)

$\begin{array}{ll}1 & (10.0) \\ 3 & (30.0)\end{array}$

$21 \quad(48.8)$

0.02

$4 \quad(40.0)$

0.02

13

(30.2)

income

$5 \quad(7.5) \quad 1 \quad$ (7.1)

2 (20.0)

$7 \quad(16.3)$

0.48

$2 \quad(4.7)$ 
Table 2. Number and percentage of responses from 2015 National Survey: Radiotherapy for metastatic breast cancer in Mexico (continued)

\begin{tabular}{|c|c|c|c|c|c|c|c|c|c|c|c|}
\hline \multirow[t]{3}{*}{ Question surveyed } & \multicolumn{11}{|c|}{ Type of medical practice } \\
\hline & \multicolumn{2}{|r|}{ All } & \multicolumn{3}{|c|}{ Private } & \multicolumn{3}{|c|}{ Public } & \multicolumn{3}{|c|}{ Private and Public } \\
\hline & $\mathbf{n}$ & $(\%)$ & n & $(\%)$ & $\stackrel{p}{\text { value }^{\dagger}}$ & $\mathrm{n}$ & $(\%)$ & $\stackrel{p}{\text { value }^{\dagger}}$ & $\mathbf{n}$ & $(\%)$ & $\stackrel{p}{\text { value }^{\dagger}}$ \\
\hline \multicolumn{12}{|l|}{$\begin{array}{l}\text { Time for initiation } \\
\text { of treatment* }\end{array}$} \\
\hline Less than one week & 35 & $(51.5)$ & 12 & $(85.7)$ & \multirow{4}{*}{$<0.001$} & 5 & $(50.0)$ & \multirow{4}{*}{0.91} & 18 & $(40.9)$ & \multirow{4}{*}{0.09} \\
\hline 8-15 days & 19 & $(27.9)$ & 2 & $(14.3)$ & & 3 & $(30.0)$ & & 14 & $(31.8)$ & \\
\hline $15-30$ days & 10 & (14.7) & 0 & $(0.0)$ & & 1 & (10.0) & & 9 & $(20.5)$ & \\
\hline Greater than one month & 4 & $(5.9)$ & 0 & $(0.0)$ & & 1 & $(10.0)$ & & 3 & $(6.8)$ & \\
\hline \multicolumn{12}{|l|}{$\begin{array}{l}\text { Most frequent indication to } \\
\text { prescribe palliative } \\
\text { radiotherapy* }\end{array}$} \\
\hline Bone metastasis & 55 & $(80.9)$ & 11 & $(78.6)$ & \multirow{4}{*}{ N/A } & 8 & $(80.0)$ & \multirow{4}{*}{ N/A } & 36 & $(81.8)$ & \multirow{4}{*}{$\mathrm{N} / \mathrm{A}$} \\
\hline Brain metastasis & 13 & $(19.1)$ & 3 & $(21.4)$ & & 2 & $(20.0)$ & & 8 & $(18.2)$ & \\
\hline Skin metastasis & 0 & $(0.0)$ & 0 & $(0.0)$ & & 0 & $(0.0)$ & & 0 & $(0.0)$ & \\
\hline Other & 0 & $(0.0)$ & 0 & $(0.0)$ & & 0 & $(0.0)$ & & 0 & $(0.0)$ & \\
\hline \multicolumn{12}{|l|}{$\begin{array}{l}\text { Most frequent schedule for } \\
\text { brain metastasis* }\end{array}$} \\
\hline $30 \mathrm{~Gy}$ in 10 fractions & 56 & $(82.4)$ & 10 & $(71.4)$ & \multirow{5}{*}{0.32} & 8 & $(80.0)$ & \multirow{5}{*}{0.71} & 38 & $(86.4)$ & \multirow{5}{*}{0.24} \\
\hline 20 Gy in 5 fractions & 7 & $(10.3)$ & 2 & $(14.4)$ & & 2 & $(20.0)$ & & 3 & $(6.8)$ & \\
\hline 2 Gy fractions for $30-50 \mathrm{~Gy}$ & 1 & $(1.5)$ & 1 & $(7.1)$ & & 0 & $(0.0)$ & & 0 & $(0.0)$ & \\
\hline One 8 Gy fraction & 0 & $(0.0)$ & 0 & $(0.0)$ & & 0 & $(0.0)$ & & 0 & $(0.0)$ & \\
\hline Other & 4 & $(5.8)$ & 1 & $(7.1)$ & & 0 & $(0.0)$ & & 3 & $(6.8)$ & \\
\hline \multicolumn{12}{|l|}{$\begin{array}{l}\text { Most frequent schedule for } \\
\text { bone metastasis* }\end{array}$} \\
\hline $30 \mathrm{~Gy}$ in 10 fractions & 43 & $(64.2)$ & 9 & $(69.2)$ & \multirow{5}{*}{0.92} & 5 & $(50.0)$ & \multirow{5}{*}{0.17} & 29 & $(65.9)$ & \multirow{5}{*}{0.52} \\
\hline 20 Gy in 5 fractions & 10 & (14.9) & 1 & $(7.7)$ & & 4 & $(40.0)$ & & 5 & $(11.4)$ & \\
\hline 2 Gy fractions for $30-50 \mathrm{~Gy}$ & 0 & $(0.0)$ & 0 & $(0.0)$ & & 0 & $(0.0)$ & & 0 & $(0.0)$ & \\
\hline One 8 Gy fraction & 10 & (14.9) & 2 & (15.4) & & 1 & (10.0) & & 7 & $(15.9)$ & \\
\hline Other & 4 & $(6.0)$ & 1 & $(7.7)$ & & 0 & $(0.0)$ & & 3 & $(6.8)$ & \\
\hline \multicolumn{12}{|l|}{$\begin{array}{l}\text { Percentage of patients who } \\
\text { are satisfied with } \\
\text { treatment* }\end{array}$} \\
\hline$\geq 90 \%$ & 37 & $(54.4)$ & 9 & $(64.3)$ & \multirow{4}{*}{0.63} & 4 & $(40.0)$ & \multirow{4}{*}{0.47} & 24 & $(54.5)$ & \multirow{4}{*}{0.23} \\
\hline $75-89 \%$ & 27 & $(39.7)$ & 5 & $(35.7)$ & & 6 & $(60.0)$ & & 16 & $(36.4)$ & \\
\hline $50-74 \%$ & 4 & $(5.9)$ & 0 & $(0.0)$ & & 0 & $(0.0)$ & & 4 & $(9.1)$ & \\
\hline$<50 \%$ & 0 & $(0.0)$ & 0 & $(0.0)$ & & 0 & $(0.0)$ & & 0 & $(0.0)$ & \\
\hline $\begin{array}{l}\text { Percentage of improvement } \\
\text { of symptoms* }\end{array}$ & & & & & & & & & & & \\
\hline$\geq 90 \%$ & 25 & $(36.8)$ & 4 & $(28.6)$ & & 4 & $(40.0)$ & & 17 & $(38.6)$ & \\
\hline $75-89 \%$ & 31 & $(45.6)$ & 7 & $(50.0)$ & & 4 & $(40.0)$ & & 20 & $(45.5)$ & \\
\hline $50-74 \%$ & 11 & (16.1) & 2 & (14.3) & 0.23 & 2 & $(20.0)$ & 0.94 & 7 & $(15.9)$ & 0.58 \\
\hline$<50 \%$ & 1 & $(1.5)$ & 1 & $(7.1)$ & & 0 & $(0.0)$ & & 0 & $(0.0)$ & \\
\hline
\end{tabular}

*For metastatic breast cancer only. Bold: statistically significant difference. ${ }^{\dagger} \mathrm{P}$-value corresponds to the 4-to-5-sample test for equality of proportions without continuity correction compared with the 'All' participants group.

lack of facilities represented an issue in $18 \%$ of cases, and the waiting time was a problem for $5 \%$.

\section{Completed radiation treatment}

Over $80 \%$ of the physicians surveyed reported that more than $90 \%$ of patients completed radiotherapy treatment. Only $3 \%$ reported that less than half of their patients completed the proposed therapy.

More than one-third of physicians reported that the main reason for abandoning the treatment was financial. The daily commute was the second reason for abandoning treatment ( $26 \%$ of cases). According to 
almost $20 \%$ of the physicians, these patients did not end radiation therapy, but instead sought another option. Only $9 \%$ of those surveyed reported that patients stopped treatment because of a poor understanding of the purpose of the treatment and its importance.

\section{From a financial perspective, what is the principal difficulty in providing treatment?}

Exclusively considering the financial component, 51\% of radio-oncologists reported that patients experienced difficulties in obtaining radiotherapy due to treatment costs. Cost of treatment was the most important factor for private practice ( $86 \%$ ), whereas lack of resources for food and lodging (30\%) and loss of income ( $40 \%)$ were the most frequent factors reported by specialists from public practice. Statistical significance was observed for both the private $(p=0.02)$ and public type of practice $(p=0.02)$.

\section{Time for initiating radiation treatment}

According to those physicians surveyed, more than half of the patients started palliative treatment with radiotherapy within one week after diagnosis. For $6 \%$ of the patients, the initiation of treatment required a waiting time of more than one month. When comparing type of practice, the treatment was found to start sooner for private compared to public practice, and was properly timed in $86 \%$ of patients in private practice and in $50 \%$ of those who were attended by public hospitals. Statistical significance was noted for private practice $(p<0.001)$ compared with the entire population surveyed.

\section{Reasons to prescribe palliative radiotherapy and schedules for brain and bone metastasis from breast cancer}

For $81 \%$ of radio-oncologists, the site of metastasis more frequently radiated was bone. Only $19 \%$ reported that brain metastases were the most frequently radiated. For bone metastasis, $64.2 \%$ of radio-oncologists more frequently recommended 30 Grays (Gy) in a 10 -fraction schedule. In total, $15 \%$ of radio-oncologists more frequently recommended $20 \mathrm{~Gy}$ in a five-fraction schedule, and $15 \%$ prescribed 8 Gy in one fraction. Only $6 \%$ of radio-oncologists preferred nonconventional schedules for bone metastasis. No statistically significant differences were observed between the groups.
The most common schedule for brain radiotherapy was $30 \mathrm{~Gy}$ in 10 fractions ( $82 \%$ of radio-oncologists). Only $7 \%$ of radio-oncologists in private practice still prescribed schedules consisting in 2 Gy per fraction up to a total dose of $30-50 \mathrm{~Gy}$. Only $6 \%$ of radiooncologists used nonconventional schedules for brain metastasis.

\section{Proportion of patients who are satisfied with radiotherapy}

In private practice, $64 \%$ of radio-oncologists perceived that over $90 \%$ of their patients were satisfied with radiotherapy compared to only $40 \%$ of patients of radio-oncologists in public practice. In addition, more than $90 \%$ of radio-oncologists who treat patients in both types of practice perceived that at least $75 \%$ of their patients are satisfied with the radiotherapy received. No significant differences were observed between the groups.

\section{Improvement of symptoms with radiation therapy for palliative care}

More than $80 \%$ of radio-oncologists considered that at least $75 \%$ of their patients had some improvement of symptoms after palliative radiotherapy. In addition, almost $40 \%$ of radio-oncologists reported that their patients had $>90 \%$ improvement of symptoms. Radio-oncologists from public practice reported higher improvement of symptoms (40\%) in their patients than radio-oncologists from private practice (29\%). In the private type of practice, $7 \%$ of radio-oncologists considered that their patients had $<50 \%$ improvement in symptoms. No significant differences between types of practice were observed (Table 2 ).

\section{DISCUSSION}

To the best of our knowledge, this is the first study aimed at quantifying the main issues faced by patients and specialists for receiving and providing appropriate radiation treatment for metastatic BC in Mexico. We analyzed, from the perspective of the radio-oncologist, the frequency of treating $\mathrm{BC}$, the proportion of patients who required palliative therapy, the difficulties with initiating and ending radiation therapy, and the protocols used for primary and metastatic tumors. 
Breast cancer mortality in Mexico has increased from $58 / 100,000$ women in 1998 to $67 / 100,000$ in 2008. As of $2006, B C$ was the leading cause of cancer death in women, representing a shift from cervical cancer and accounting for $14 \%$ of all cancer cases ${ }^{5,6}$.

Radiotherapy is a key component in the treatment of $\mathrm{BC}$ and is typically indicated after mastectomy and for the treatment of metastasis, including bone, brain, and soft-tissue metastasis ${ }^{9}$. From our survey, we were able to determine that, similar to what is observed in a number of high-income countries, radio-oncologists in Mexico primarily treat $\mathrm{BC}$, which is the most frequent tumor location that requires radiation therapy $^{13}$. Approximately $83 \%$ of new BC cases require radiation therapy ${ }^{10}$. In addition, $22 \%$ of bone metastases are derived from breast tumors. In fact, BC is one of the leading causes of bone metastases, varying in prevalence from 47 to $85 \% 5$. Our survey indicated that more than $60 \%$ of radio-oncologists' practice involves breast tumors.

Palliative radiation treatment is highly important for BC therapy in Mexico, especially considering that $11 \%$ of cases present with metastasis at diagnosis. The availability of palliative care services has increased since 1970, when the first service was established at the Instituto Nacional de Cancerología, the largest institute for cancer treatment in Mexico. In 2007, the first official policies for palliative care were published. In 2009, based on international guidelines, recommendations for BC management, including guidelines for pain control and palliative care, were also published $^{1}$.

As expected, patients treated by private physicians initiate radiotherapy more quickly, typically within $<15$ days. In contrast, delays are more common in patients attended in public hospitals, where up to $10 \%$ start treatment after one month.

Despite the national insurance coverage for $\mathrm{BC}$ treatment, physicians report that cost is still the determining factor for receiving radiotherapy. The lack of facilities is also an issue, even for radio-oncologists in private practice, who reported that it is an important issue in approximately $20 \%$ of cases. According to the National Commission of Nuclear Security and Safeguards (NCNSS), there are 83 registered radiotherapy centers in Mexico, 45 public and 38 private. There are only six centers for radiotherapy training, and only two of them are specialized in treatment for children. Although Mexico has 23 public centers for cancer treatment located in several states of the country, the characteristics of the equipment vary between centers, including different dosimeters and simulators and differences in the treatment planning capacity. Our survey suggests that patients treated in private practice are more likely to follow treatment than those attended in public hospitals (100 vs. $80 \%$ ).

As mentioned previously, more than $20 \%$ of bone metastases are derived from breast tumors. Accordingly, we found that the most frequent site of metastasis for radiation therapy is bone.

Breast cancer is the second cause of brain metastasis. Up to one-third of BC patients exhibit brain metastasis during the course of the disease ${ }^{14}$. In addition, $3 \%$ of patients with local disease, $8 \%$ of patients with regional disease, and $13 \%$ of patients with stage IV disease will develop central nervous system involvement ${ }^{15}$. Radiation therapy is essential for treating brain metastasis, requiring the most sophisticated procedures, high costs and capacity of human resources, equipment, and an increased number of treatment hours. In our study, approximately $20 \%$ of radio-oncologists reported brain metastasis as the most frequent site radiated.

To offer effective palliative treatment, radiation therapy must be administered shortly after diagnosis to reduce symptoms and avoid additional damage to the patient, which typically occurs in association with spine or brain metastasis. In our study, we found that more than half of patients started palliative care with radiotherapy within one week after diagnosis, and this treatment begins sooner in private than in public practice.

In general, there is a trend to reduce the time of treatment using radiation in palliative care. Shortened palliative treatment schemes may confer a similar effect as longer schemes ${ }^{16}$ and may allow increasing the efficiency of treatment and the number of patients treated in radiotherapy units. We observed that more than $90 \%$ of the radio-oncologists use the standard short schedules for brain and bone metastasis. Overall, the schedules are shorter for bone than for brain metastasis. 
Comparatively, a larger proportion of patients attended in private practice appeared to be satisfied with radiotherapy than patients treated in public hospitals. While this may largely be due to differences in treatment schedules, other factors, such as longer waiting times to start treatment and more financial barriers, seem also important for those patients attended in public practice.

Regarding the improvement of symptoms using radiation therapy, the physicians from public practice reported higher rates than those from private practice. Although it is possible that patients attended by private physicians may have higher expectations or less tolerance to pain, further research on this issue is necessary.

According to recent reports, 30 of the 32 States in Mexico have at least one radiotherapy unit ${ }^{13}$. There are 102 megavoltage machines in Mexico (including linear accelerators and cobalt-60 machines), which means 1.08 megavoltage machines per million inhabitants, with an estimated demand of 149 for the year 2002. Although we did not ask about affiliation with national healthcare subsystems, according to the CNSNS, there are 92 linear accelerators for radiation therapy in Mexico, including 26 belonging to the Ministry of Health, 17 to the Instituto Mexicano del Seguro Social (IMSS), and two to the Instituto de Seguridad y Servicios Sociales del Estado (ISSSTE) ${ }^{17}$.

The exact number of radiation oncologists in the country is unknown, although one study estimated that the total number of certified radiation oncologists in Mexico was 200 in $2013^{13}$. Studies from Mohar, et al. reported 58 radiation oncologists affiliated with the Ministry of Health in $2009^{5}$. It has been estimated that nearly 4-7 radio-oncologists are required per 1,000,000 inhabitants. Thus, between 200 and 350 specialists are required, considering only patients who do not have access to social security (approximately 45 million people are covered by the Ministry of Health).

In addition, the mean number of treated patients per year per facility in Mexico is 829 . Thus, Mexico does not meet the World Health Organization criteria for sufficient radiation therapy protocols ${ }^{5}$. Additionally, only $15 \%$ of cases received conservative surgery plus radiotherapy in Mexico, which was partially due to long waiting times to access radiotherapy $(\sim 60 \mathrm{cal}-$ endar days) ${ }^{5}$.

Our survey also analyzed factors considered important to improve radiotherapy treatment in Mexico. As it was an open question in the survey, we do not present numeric data. Most respondents indicated that increased specialized equipment as well as more radio-oncologists, technicians, and physicists are required. Other suggestions included reduction in treatment costs and improved processes to acquire equipment, such as lineal accelerators, and more interactions with other oncologic specialties.

Our study has several limitations. First, it relies on the opinion of specialists and, unfortunately, the response rate was limited. However, the questions were designed to obtain an approximate, rather than a precise estimation of current treatment patterns. Furthermore, we did not request information related to the specific place in which the physician practiced or to the corresponding institution of the health system (Ministry of Health, IMSS, or ISSSTE), which limits our ability to interpret the differences across public institutions. In addition, our study is limited because our survey was offered in English to facilitate administrative procedures with the IRB, leading to a selection bias in physician response. However, for any medical specialty in Mexico, including in radiation therapy, a sufficient level of understanding of written English is required.

In conclusion, using a survey targeted at physicians, we identified some of the most important issues faced by patients and specialists related to radiation therapy in Mexico. We also determined some serious difficulties to start, maintain, and complete therapy, the radiotherapy schedules commonly used, and perception of physicians about patient satisfaction. These results provide important insight for future policies related to radiation therapy for $\mathrm{BC}$ in Mexico.

\section{ACKNOWLEDGMENTS}

NA was supported by Instituto Nacional de CancerologíaMéxico (INCan). DP is financially supported by Consejo Nacional de Ciencia y Tecnología (CONACYT) and the Fundación México en Harvard from the program, "Postdoctoral Fellowships in the Sciences at Harvard University: Mexico." Felicia Knaul was director of the Harvard Global Equity Initiative (HGEI) when this survey was undertaken. NA led this 
project during her fellowship as visitor scientist in HGEI. HC belonged to HGEI when this project was carried out.

The authors thank Clementina Castro for her critical review of this manuscript.

\section{REFERENCES}

1. Chávarri-Guerra Y, Villarreal-Garza C, Liedke PE, et al. Breast cancer in Mexico: a growing challenge to health and the health system. Lancet Oncol. 2012;13:e335-43.

2. Bray F, Ren J-S, Masuyer E, Ferlay J. Global estimates of cancer prevalence for 27 sites in the adult population in 2008. Int J Cancer. 2013;132:1133-45.

3. Stewart BW, Wild CP. World Cancer Report 2014. Available at: http://publications.iarc.fr/Non-Series-Publications/World-Cancer-Reports/World-Cancer-Report-2014.

4. Atun R, Jaffray DA, Barton MB, et al. Expanding global access to radiotherapy. Lancet Oncol. 2015;16:1153-86.

5. Mohar A, Bargalló E, Ramírez MT, Lara F, Beltrán-Ortega A. [Available resources for the treatment of breast cancer in Mexico]. Salud Publica Mex. 2009;51(Suppl 2):s263-9.

6. Knaul FM, Nigenda G, Lozano R, Arreola-Ornelas H, Langer A Frenk J. Breast cancer in Mexico: a pressing priority. Reprod Health Matters. 200816:113-23.

7. Knaul FM, Nigenda G, Lozano R, Arreola-Ornelas H, Langer A, Frenk J. [Breast cancer in Mexico: an urgent priority]. Salud Publica Mex. 2009;51(Suppl 2):s335-44.
8. Offersen BV, Boersma LJ, Kirkove $C$, et al. ESTRO consensus guideline on target volume delineation for elective radiation therapy of early stage breast cancer. Radiother Oncol. 2015 114:3-10.

9. Walsh SM, Lowery AJ, Prichard RS, McDermott EW, Evoy D, Geraghty J. Postmastectomy radiotherapy: indications and implications. Surgeon. 2014;12:310-15

10. Barton MB, Frommer M, Shafiq J. Role of radiotherapy in cancer control in low-income and middle-income countries. Lancet Oncol. 2006; 7:584-95

11. Nigenda G, Caballero M, González-Robledo LM. [Access barriers in early diagnosis of breast cancer in the Federal District and Oaxaca]. Salud Publica Mex. 2009;51(Suppl 2):s254-62.

12. Comparing more than two proportions. Boston University Schoo of Public Health; Available at: http://sphweb.bumc.bu.edu/otlt/ MPH-Modules/BS/R/R6_CategoricalDataAnalysis/R6_Catego ricalDataAnalysis6.html [Accessed 2016 Jan 29].

13. Poitevin-Chacón A, Hinojosa-Gómez J. Patterns of care of radiotherapy in México. Rep Pract Oncol Radiother. 2012;18: 57-60.

14. Kong W, Jarvis C, Mackillop WJ. Estimating the need for palliative radiotherapy for brain metastasis: a benchmarking approach. Clin Oncol (R Coll Radiol). 2015;27:83-91

15. Gil-Gil MJ, Martinez-Garcia M, Sierra A, et al. Breast cancer brain metastases: a review of the literature and a current multidisciplinary management guideline. Clin Transl Oncol. 2014;16: 436-46.

16. Dixon $P$, Mackillop W. Could changes in clinical practice reduce waiting lists for radiotherapy? J Health Serv Res Policy. 2001;6: 70-7.

17. Comisión Nacional de Seguridad Nuclear y Salvaguardias (CNSNS). Available at: http://www.cnsns.gob.mx/ [Accessed 2016 Nov 29]. 